\title{
10
}

\section{Using the Internet to implement support for distributed decision making}

\author{
A. R. Dennis
}

Terry College of Business, University of Georgia

Dept of Management, Athens GA 30602, USA, Tel:+1 (706) 542

3743, Fax:+1 (706) 542 3743, Email: adennis@uga.cc.uga.edu

F. Quek

London School of Economics and Political Science

Dept of Information Systems, Houghton Street, WC2A 2AE, UK,

Tel:+44 (0171) 955 7403, Fax:+44 (0171) 9557565 ,

Email:f.k.quek@lse.ac.uk

\section{S. K. Pootheri}

Dept of Mathematics, University of Georgia

Athens GA 30602, USA, Email: sridar@math.uga.edu

\begin{abstract}
This paper addresses implementation from the point of view of DSS construction and installation, and highlights the challenges faced when developing DSS in light of rapidly changing business environment and technological advances. In view of the tremendous interest in the Internet, the paper suggests that the Internet has inherent characteristics that are wellsuited to supporting distributed work and distributed decision making. The paper then presents TCBWorks as a first generation DSS built for the Internet, and hopes that through sharing the experiences and lessons learnt, the potential and pitfall of this technology and its fit to distributed decision making can be explored, thereby guiding further research in this area.
\end{abstract}

\section{Keywords}

Internet, Distributed Decision Making, TCBWorks, Web-based Groupware, DSS Implementation, Web-based technologies, Communicative Competence

\section{INTRODUCTION}

Decision Support Systems (here taken in the broadest sense) are technological artifacts which are created from and with computer-based technology (Silver, 1991). As software engineered systems, they draw heavily upon the wide range of computer science and management science 
technologies. DSS developers, not unlike any software developers, will use programming languages, development tools, specialised designed tools or "DSS Generators" (Sprague, 1980), or embed other technologies such as graphics, dialog management, and model management. This paper focuses on DSS technologies for the implementation ${ }^{*}$ of the designed solution, that is the construction and installation of such systems (Silver, 1990; Fenton \& Hill, 1993). Though the choice of the development tool or system plays a key role in determining the success of any system such as a DSS (Silver, 1990), their implementation has become ever more difficult.

The world we live in today is in constant and rapid change, reflecting the dynamism on the one hand, and the chaos and confusion that they create on the other. These rapid changes in business and technological advances mean that DSS need to be developed and implemented quickly enough to respond to decision maker's changing needs. DSS as a class of computerbased solutions have their own unique characteristics (Kivijarvi and Zmud, 1993) which neccesitate their development being different from traditional system development life cycle (SDLC) approach used for transaction processing systems. Because of the semistructured or unstructured nature of problems addressed by DSS, managers' perceived needs for information will change and so the DSS must also change (Turban, 1990, p. 158). This has the inadvertent effect of resulting in systems with short life-spans that lose their utility quite rapidly, and may actually become a competitive disadvantage by locking organizations into decision processes that cannot change to meet new requirements.

Traditional SDLC approach has given way to Prototyping and Rapid Application Development (RAD). Also, unlike in the past where a programming language or development tool can have a shelf life of $n$ years, technological advances have in effect, introduced newer versions rapidly. These days, a version difference can mean a total reskilling and retooling. Big tools developers like Microsoft even introduces the policy that version support is limited to just 1 year from the introduction of the new version. As such, developers constantly have to deal with the dilemma of deciding whether revisions to systems (maintenance or enhancements) should be completely rewritten in the latest version, or with another tool or language. This dichotomy of innovation on one end and continuity on the other, highlights the challenges faced when developing DSS in a rapidly changing business environment and technological advances (Benamati et al, 1995). For DSS to be viable and popular (i.e., cost, speed of development, scalability and ease of maintenance), the choice of the right development strategy and tool become ever more important to determine their success.

There is no doubt that the Internet, and in particular, the World Wide Web, has become an important form of communication technology. It is threatening to invade our homes just like what personal computers, telephones and televisions have already done. While personal computers offer computing power, the Internet offers online computing and access to information on a scale unimaginable even a few years before.

From the point of view of technological advances, the Internet technology is compelling. It seems to be going from strength to strength, and looks like being the backbone of a whole new way of computing. Web-based applications are sprouting out everywhere, and we are beginning to see a new generation of systems built for the Internet, with major hardware and software companies pledging support and jumping onto the Internet bandwagon.

\footnotetext{
*For a fuller discussion on Implementation, see Daellenbach (1994)
} 
From the point of view of organisational decision making, the Internet is also compelling. "Almost every time there is a genuinely important decision to be made in an organization, a group is assigned to make it -or at least to counsel and advise the individual who must make it." (Hackman and Kaplan, 1974). Much research on organisational decision making has examined the role of computer-based systems in supporting group decision making, not only the traditional DSS-style decision support function, but also the communication needs of groups, whether they meet together in the same room at the same time, or are distributed groups whose members work in different locations at different times (Jessup and Valacich, 1993; Johnson-Lenz, 1992; Nunamaker et al, 1991). The Internet, and the Web in particular, may be the ideal technology to support the communication needs of distributed groups.

Our goal in this paper is to share our experiences in developing and using TCBWorks, a first generation Web-based groupware system that can support distributed decision making. TCBWorks is designed to be used in any place, at any time, whether participants meet together in a special purpose computer-supported meeting room or work across the Web from different locations in different organisations.

\section{THE INTERNET}

The Internet is basically one big global network of computer networks. Once you connect your PC to the Internet, it can talk to any other computer in the world that is also connected with. Synonymous with the Information Superhighway, the Internet came into existence in 1969 as ARPANet, a US Defense Department network supporting military research (Krol, 1992). It was developed to satisfy the need for researchers at different geographical locations to be able to communicate with each other on a more rapid basis than before (Uhlig, Farber and Bair, 1979). However, connection only became more easily available and open to a wider audience in 1982 when the National Science Foundation developed NSFNet which was the precursor to finally enabling everyone access to the network.

There are two directly opposing views of the Internet. As stated in an article in Personal Computer World (Jan, 1996): "One is that it is full of junk and porn, stuffed full of useless information no-one needs and a hang-out for losers and perverts. The other is that it offers people a new golden age of information that will liberate them from industrial slavery". Both of these views are somewhat distorted to the extreme. The Internet certainly is a mass media distribution mechanism for a variety of information, software, entertainment and commerce. Not only can it open up the world where you can be put in touch with people around the world, but it has the potential to bring a richer medium of communication that can contain audio, video, text, graphics, modelling etc.

The Internet holds the promise of a technology that can rival the significance of the telephone and television that connects the world. The advent and availability of personal computers in the mid 1970s, once strictly in the domain of government agencies, researchers and scientists, have been the major factors in changing the way we work today and in the future. Their pervasiveness in homes and offices today, across all age groups and occupational levels, have certainly influenced the development of interactive systems like DSS as prior to this, many of the office applications of computers lie in non specialist uses (Newman, 1987). 
Telecommuting, the technology of bringing the work to the workers (Kelly and Gordon, 1986) is already widespread, especially with doctors, lawyers, sales people and researchers. And there is also the emergence of Groupware technology, and CSCW as a field of study. In essence, we are seeing the convergence of a number of technologies which are heralding in an era of distributed technology for distributed work. Despite all these potential and possibilities, there are still a number of major issues to be addressed: security, electronic payment protocols, bandwidth constraints, standards, governance etc. Until they are dealt with effectively, the hype and the scepticism will continue to cast a shadow of doubt over the Internet.

Existing office computer systems do not always offer the flexibility required to meet the needs of today's user. The problem is that people need to have access to organisational information in a relatively unstructured manner, access from any location. Noam (1995) highlighted a reversal in the historic direction of information flow: "In the past, people came to the information, which was stored at the university. In the future, the information will come to the people, wherever they are". And this is where the authors believe that the Internet can hold the key in revolutionising the way businesses are conducted, offices communicate, workers interact and systems are developed and use (Quek and Tarr, 1996)

By far, one of the best example of using distributed technology to support distributed work is e-mail. It has not only become popular in the academic and research world, but is fast becoming a standard practice in the business world (Crawford, 1982). It is the choice of managers (Markus, 1994) and even the home as it is much faster and cheaper than faxing or snail mail (conventional mail). It is also capable of sending documents together with the mail, providing a much richer medium (Rice and Shook, 1990; Lee, 1994).

\section{SUITABILITY OF THE INTERNET FOR DSS IMPLEMENTATION}

Technological advances and the myriad of development tools available today are still beset with issues of incompatible machines, platforms and operating system, on top of costly proprietary systems. The Internet has offered a new dimension of appreciating and evaluating the opportunities for completely new ways of doing things. As researchers, the authors feel that it is important to explore this technology to frame and extend the discussion about the role of the Internet in the future of DSS, and identify some meaningful and effective use.

\subsection{Addressing Implementation Challenges}

The construction and installation of DSS are not everyday occurrences that organisations and individuals can commit themselves to easily. They can not only be costly undertakings but are also highly specialised applications. The Internet has the potential to address some of these implementation challenges. Not too long ago, client-server technology was touted as the technology of choice for distributed organisations as a means to improve productivity, reporting and decision making. Likewise, Groupware technologies were held in the same breath to provide the mechanism for co-ordination and control of group work. Today, both of these are seriously under threat by the Internet development, and may not face up to the more open Internet which provides an inexpensive alternative. The following are some implementation challenges that can be addressed by using Internet technology: 
- Distributed technology - Because the Internet was borne out of a US military project to address the concern of a nuclear strike wiping out a single-site computer system, it can be a very secure and reliable way of providing distributed working.

- "Open" Technology - Development tools and languages such as Java, provide development of applications which are independent of platform and operating system, and be able to run client application no matter where you are or what machine is being used. Unlike previous "Open" systems ventures such as those between IBM and Apple (1991) to create Taligent, an operating system built on object oriented technology, the push for "Open Systems" has not seen the kind of success that the Internet has achieved in a relatively short period of time. In effect, it is becoming the catalyst for vendors, developers and users to work towards more "open" technology.

- Promote reuse - SunSoft's Java scripting language promises a new generation of Internet applications that is an example of a true application of object technology. This promotes reuse of objects like assembly blocks when constructing applications.

- Accessibility - Even though the Internet has been around since 1969, it did not exactly take off until 1992 when the World Wide Web (WWW) came about, a collection of servers working together to form a graphically-based hypertext network. It is through this user interface that the Internet suddenly become accessible to a global community without barriers of language, culture or geographical distinction.

- Availability - Today, the Internet is often quoted as being made up of 50,000 networks, four million computers and 20-40 million users. Its exponential growth is expected to continue, with office networked computers expected to have Web access, people buying affordable Internet boxes, and home computers hook up via Internet Service Providers.

- Distributed Resources - There is no need to rely on dedicated resources from one single source. The Internet technology actually addresses the problem of different platforms and operating systems, and had successfully developed protocols to enable different networks to communicate with each other. One example of an overwhelming success of this technology is e-mail.

- Development of Intranet - The private use of Internet technology within organisations are known as Intranets, which will provide a seamless application platform for users both inside and outside the organisation.

- Distribution of System/Installation, Version Upgrade and Maintenance - Unlike groupware or other current technologies which have the problem of availability of client program, the right version for a platform and operating system, and keeping it up-to-date, Internet programs such as Java applets need little client-side maintenance. Because applets are downloaded from the server before executing on the client, the current version is automatically downloaded. They are also highly portable, thus reducing both maintenance and time-to-market for multiplatform applications.

\subsection{Current Research Interest}

There has been a growing interest in distributed systems such as distributed GSS/GDSS, and this can be seen as a natural progression from earlier work in GDSS/GSS and their applications such as Decision Conferencing and Electronic Meeting Systems (EMS) in the late 1980s and early 1990s (Jelassi et al, 1992). Most of these research focus on decision room 
environment where a group of participants meet face-to-face in the same room, working on a common task (Dase et al, 1995; Dennis et al, 1990/91; and Stohr and Konzynski, 1992).

Dase et al (1995) thus suggested that current research framework developed for GSS/GDSS (McGrath 1984; Dennis et al, 1988; and Nunamaker et al, 1993) must be extended or adapted to deal with new issues in contrast to co-located participants/decision makers (Gallupe and DeSanctis, 1988). As such, DGSS/DGDSS is relatively new and there is little theoretical or empirical research on this topic. In short, the multiple decision maker aspect (Huber, 1984), the geographically dispersed aspect (Hiltz and Turoff, 1992) and the enabling technologies aspect (Fellers et al, 1995 and Bhargava et al, 1995) raise many new questions, challenges and opportunities in research and application areas.

DGSS/DGDSS is gaining popularity not because of advances in long distance communication systems and satellite transmissions, but because of the Internet's mass appeal. Fellers et al (1995) argues that the innovation in using systems (technologies) comes not from their existence, but from their increasing availability to the general public. The Internet growth points to a new application development trend which provides exciting possibilities for distributed applications. Despite its early days, the Internet technology can and should be exploited to further the goals of DSS (Bhargava et al, 1995). One such implementation is DecisionNet (Bhargava et al, 1995), a repository of decision support and modelling technologies that also allow these technologies to be used interactively over the WWW. Another example is the research at Humboldt University on developing "method servers", WWW-based servers of computational services such as algorithms and solvers (Krishnan et al, 1995). DecisionWeb is a system designed to be a "front-end" for traditional problem-solving meetings to enable a group to list, categorize and prioritize ideas asynchronously via the Internet prior to a meeting (Danyi, 1994). Other uses of the Web include providing web pages of topic specific information and resources which have proved to be popular, for example ISWorld Net DSS (Power et al, 1995) and Groupware Central (http://www.cba.uga.edu/group ware/groupware.html).

\subsection{Addressing Systems for providing Decision Support}

It can be said that much of the mainstream research into DSS tend to be technologically focused, and still lacks the promise that remain true to what DSS aims to do, that of providing support for ill-structured problems (Sprague and Carlson, 1982). Humphreys and Nappelbaum (1995) argued that there is still an almost complete absence in practical applications at top management level of interactive computer-based systems based on traditional DSS and IS design methodologies (Lederer and Sethi, 1988). A decade earlier, Humphreys (1984) had already raised this concern, and advocated for the development of "techniques for the psychological validation of the decision makers' own problem structuring language than to try to invent a universal problem structuring language that will have to be taught from scratch to high level decision makers". Alter (1979) perhaps provides the best explanation for this state of affairs: simple DSS are easier to understand, implement, control and modify than complicated DSS. The advent of personal computing had led to the natural application of computing power and technology to managerial needs such as in decision making, by making new discoveries in decision methods or decision technology and applying them through creating tools that decision makers might find helpful. Most of these efforts have been in 
decision analysis and other forms of decision modeling and human information processing through interactive use of computers, electronic storage media, and electronic communications and information display.

This evolution of DSS, which focuses on the development of technologically supported means of collecting, managing, and displaying information that might be useful in decision situations (Sprague, 1980; Steeb and Johnston, 1981; Stodolsky, 1981; Turoff and Hiltz, 1982; Vogel and Nunamaker, 1988), sadly, also reflected the failure of the traditional DSS/IS approach with complex DSS (Ginzberg, 1975; Manley, 1975; Lucas, 1976; Alter, 1979). GDSS soon came into the limelight, stemming in part from the rise of interest in the area of technological support for groups. Much of GDSS research has taken the view that the most fundamental activity of group decision making is interpersonal communication and hence, to improve group communication activities (DeSanctis and Dickson, 1987), resulting in the development of computer-based workbench environments which facilitate group communication.

But this popular form of DSS/GDSS research did not actually address what Kraemer and King (1988), and Phillips (1989) identified as the other approach to DSS/GDSS, of studying the decision making itself both at individual and group levels (cf. DeSanctis and Gallupe 1987), on "discovering psychological or cognitive processes of individuals and groups involved in reaching conclusions and on the sociology of small-group interaction". This second approach of providing " a problem solving environment that is group centred and is primarily intended to help managers consider uncertainty, form preferences, make judgments and take decisions" claimed Phillips, is a more superior approach. Clearly the first approach seems more feasible and less problematic to undertake research than the second, and understandably reiterates Alter's point about dealing with creating simple DSSs. It is easier to build technical aids for decision making than to paint a clear picture of what decision making is. These new technologies are widely adopted and used, but it still is not always clear whether they really improve the condition of those who use them.

\subsection{Addressing Support for Distributed Decision Making}

The body of research in DSS/GDSS and their conception of "group" may not be relevant or appropriate to study about the growing interest in supporting distributed decision making and cooperative work activities. Groups are not always small, participants come and go, their goals are neither shared nor existing, their tasks can be ambiguous, and decision making may occur in a distributed fashion (Lyytinen et al, 1994; Bannon, 1994). In their CSCW-related research, Schmidt and Bannon (1991) find "cooperative ensembles" (instead of the word "groups") are either large, or are embedded within larger ensembles. They are also often transient formations, emerging to handle a particular situation after which they are dissolve again, and their work is distributed logically, in terms of control, and involve incongruent strategies and discordant motives. There is also the notion that there is no omniscient agent in many of these situations. As such, decision making is inevitably distributed among the decision making agents who have unique situations to face, and their own sets of perspectives, goals and interests to take into account. The question is how then do we address the support for such distributed decision making? 
One approach is the integration of CSCW work into GDSS research. The former's strength is in its detailed investigation of exactly how work gets done in organizations, with a particular focus on cooperation, co-ordination and communication in work. For example, in $\mathrm{CSCW}$ research, attention has been turned towards the development of an understanding of how to support distributed decision making and cooperative work activities where people are working at "arm's length", without direct communication and without necessarily even knowing each other or knowing of each other. In such cases, people must cooperate via a more or less shared or common information space, such as a 'space' comprising data, personal beliefs, shared concepts, professional heuristics etc. (Bannon \& Schmidt, 1991), which is typical of a distributed organization.

Decision makers in a distributed environment should still have the benefit of equal time and opportunity to participate and influence the decision task at hand. Habermas's (1990) theory of communicative action advocates taking informed action through an ideal process of " $a$ forum of speakers exhibiting communicative competence" (Jackson, 1991), as opposed to reducing the stakeholders from the role of active participants to that of mere "witnesses" (Ulrich, 1988). As a basis of communicative action all participants will ideally, need to provide information about "what they know best". It is also necessary to organize, and feedback, on a distributed basis, an enhanced understanding of this collective knowledge, given that a suitable vehicle of communication could be found for this purpose.

It is the identification of this suitable vehicle of communication to support Habermas' theory which the authors believe the Internet to be the potential contribution in supporting decision making in a distributed environment. Having an effective vehicle of communication means an orientation towards the discourse within an interpretive framework where each participant's assumptions about what is (or assumed to be) commonly known within the organization can surface (McCaskey, 1988). Therefore, it is not apppropriate to presume the hegemony of the interpretations and assumptions of any particular group or stratum (Humphreys et al, 1995). We are only at the stage of dealing with the efficiency of the vehicle of communication. In this light, the authors introduce TCBWorks as an example of how using web-based technology can potentially help improve the communicative competency of distributed decision makers.

\section{USING THE WEB TO SUPPORT DISTRIBUTED DECISION MAKING}

The technology for supporting group work (on-site or distributed) has spawn the emergence of groupware and groupware technology. Many groupware systems use proprietary software and architectures running over local area networks or proprietary wide area networks to distribute information among participants. Until recently, there have been few alternatives. The recent explosion of the Web presents a new opportunity as we can now build systems and architectures that take advantage of widely adopted open standards that are available to most potential users of groupware. 


\subsection{Groupware}

Groupware is a term that is commonly used, but lacks a commonly accepted definition. In general, it is a set of hardware and software designed to help groups work together. Vendors, consultants, users, and university researchers all have used the term to refer to many different types of software, each of which support very different types of group work. This form of groupware provides two key functions to groups. First, it enables group members to generate, read, and organise information in a structured manner (Nunamaker, 1991). A weak form of this type of groupware is the usenet newsgroups, which are very good at enabling many people to generate information, but are seriously lacking in the ability to organise it. Groupware enables groups to edit, move, delete, and structure information so that it is presented in a hierarchy or map that is easy to analyse and can evolve as new information is added.

The second key function of this form of groupware is the ability for group members to rank, rate, or otherwise quantitatively analyse the relative merits of alternatives (i.e., to vote) (Nunamaker, 1991). Most groupware systems enable users to vote by ranking or rating alternatives. Some support multicriteria decision making, so that a set of alternatives can be evaluated by all members on a series of criteria (e.g., rating cars on gas mileage, acceleration, etc.). Others support more elaborate decision analysis processes. In any case, each participant enters his or her ratings, analyses, or votes, which are then combined with those of all other participants and presented to the group as a whole for further discussion.

Many companies now use this form of groupware in special purpose meeting rooms, as well as using it to enable groups to "meet" from different geographic locations. This form of groupware has also been the subject of much academic research. While it can improve the quality of group decisions in some cases, its primary benefit is time savings: research with more than 40,000 participants suggests that it can reduce the time needed to make decisions by $50-90 \%$ (Groupware Central, accessed 1995; Jessup \& Valacich, 1993; Nunamaker, 1991).

\subsection{TCBWorks: A first generation Web-Groupware system}

TCBWorks (http://tcbworks.mgmt.uga.edu:8080) is different from the "typical" discussionoriented tools now available on the Web. It is designed to enable people to interact, discuss issues, and make decisions. It can support both structured discussions and multicrieria decision making. The principal organising object in the TCBWorks is the project. A project contains all the data and processes needed to perform most group tasks. Projects are organised in a hierarchy, so that projects can contain sub-projects, sub-sub-projects, and so on. All projects contain the knowledge to be added, deleted, modified, and moved, among other functions. Each project in turn contains a set of topics. Topics contain many of the same properties as projects. They are also organised in a hierarchy (with a series of topics, subtopics, etc. within each project), and can be added, deleted, modified, and moved.

Topics in turn contain comments (short paragraphs of text) that can be added, deleted, and modified. Comments can also contain HTML tags, enabling participants to specify formatting (e.g., bold, italics, bullet lists), as well as taking advantage of all the other benefits of the web. It becomes simple to embed a graphic in a comment (provided, of course, you know the HTML syntax and the web address of the graphic). It is also simple to put a link to other web 
documents as part of a comment or a reference to more information. Comments can be anonymous or identified by the name of the contributor.

Each topic can be rated (i.e., voted on) using a set of criteria defined by the group. There are a maximum of ten criteria, each with user-defined ranges (i.e., minimum and maximum values). Each participant can enter a rating for each topic for each criterion and the system provides the mean value of the group's ratings. The topics can then be viewed in order based on their mean rating across all criteria, or on the mean rating for any one criteria.

There are four categories of users in TCBWorks:

- System Administrator: the super user can perform any function, such as to create and delete other users;

- Project Organizer: the user can create new projects and perform any function to the projects created, and also grant access to other users, delete the project, move it, etc;

- Participant: the user can only access those projects to which he or she has been granted access by a project organizer, and perform those functions permitted by the organizer;

- Observer: the user has read-only access to the projects specified by an organizer.

\subsection{Interface and Technical Design}

When the user first connects to the web server, it presents a login screen that requests for the user's name and password. Once successfully logged on, the user is presented with the Project Screen, which displays the list of projects available to the user (all projects to which the user has access). The action buttons displayed on the left of the screen change depending upon the type of user. A project organizer would have all of the buttons, except the Controls button, which is used to control access to the database (e.g., create new users). A participant has no ability to create or change a project, and therefore only have the Open and View buttons (as well as help, refresh, and exit). Not all of the buttons available to an organizer may be valid for a specific project. For example, an organizer of one project may only grant participant rights for that project (not organizer rights) to another organizer, meaning that he or she could not delete the project.

Once a project has been selected, the user clicks the Open button, and the Topic Screen is displayed. This screen displays all the topics that exist for the project and enables the user to discuss topics and add new ones. Once again, the buttons that are displayed depend upon the type of user and the rights granted by the project owner. System administrator(s), the project organizer, and any user to whom the organizer has granted organizer rights can perform any function. Participants will only have those buttons to which the organizer has granted rights.

The Topic Screen enables users to perform two major functions (aside from editing and adding topics): discussing the topics, and voting. The discussion screen displays all the comments entered under a topic, and provides an input box for participants to enter their comments. There are typically many comment from different participants under the same topic. Comments are normally displayed in the order in which they are entered, but participants also have the option to insert comments after a specific comment, simply by changing the number in the comment number box. The organizer can specify whether participants can Insert, Delete or Replace comments, as well as specifying whether comments will be anonymous or identified by the name of the contributor. 


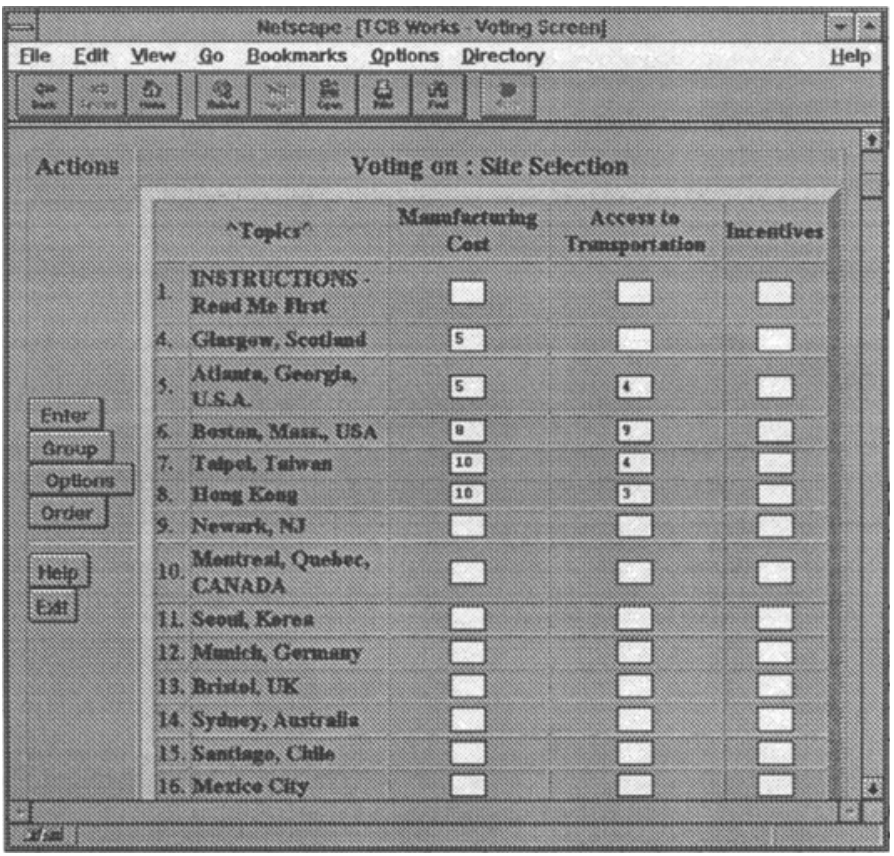

\section{Figure 1 Voting Screen}

The last major function is voting (see Figure 1). The organizer first defines the criteria that will be used (anywhere from 1-10 criteria are possible) and the scale that will be used for each (any integer range; the default is 1-10). Once these have been defined, participants can enter their opinions on each of the topics in the project in the boxes provided. Votes can be easily changed at any time and participants can move back and forth between the screen where they type their votes and the screen that displays the average vote of all participants.

The technical design of the system consists of using a series of $28 \mathrm{C}$ programs (about 30,000 lines of code) working on data in an SQL database to generate the HTML forms. These forms capture the user's commands and present information in response, and are generated on the fly. Each program in the system first generates an HTML form by reading the parameters passed by the previous program and by accessing data from a MiniSQL database. Since groupware is inherently a multi-user environment, the use of a database engine greatly simplifies programming by handling the potential concurrency problems, such as the ability to lock records to indicate to other users that someone is making a change.

The HTML forms collect the user's commands through the use of push buttons such as "add a new project". We found this approach to be very efficient. It is modular so it also reduces the complexity of the system. The Web server does not remember the previous states that it took to reach any given screen. Each transaction is a separate request. The user can get into any screen (sometimes using a bookmark) and exit at any state. Therefore, the HTML 
form that is returned with each request must contain sufficient information to tell the system all choices made by the user in the previous screen, in addition to the current request.

\subsection{Experiences using TCBWorks}

TCBWorks is currently in use by more than 100 organisations around the world. Most are headquartered in North America, followed (in order) by Europe, Australia-New Zealand, Asia, South America, and Africa. There are many ways of examining the ways in which TCBWorks is being used. One is to consider distance and time. TCBWorks was designed to be used by people working in different places and times across the Internet. However, more than half the organisations are also using it in intranets (intranets use the same network standards as the Internet e.g. TCP/IP, but are designed primarily for private use). The most common use is to support different time and place discussions within a campus or corporate office park, but it has also been used to support same time and place meetings in decision room settings and those where some meeting participants are at remote locations.

Another way of examine its use is to look at the nature of the groups and tasks. One classification is a loosely connected electronic community of users that share the same interests and many of the same goals. An example here is the Executive Operating Committee of ISWorld Net which will be elaborated in the next section. Similarly, a variety of student groups at the University of Georgia and elsewhere are using the software within their campus (i.e., an intranet) to discuss issues of interest to the students. Job-hunting, and course evaluations are typical applications. The key benefit here and in the ISWorld Net example, is the ability to hold formal and informal discussions and to share information and opinions among a groups of individuals with common interests in a more structured form than a newsgroup, listserv, or mailing list would provide.

Task forces and project teams are more tightly connected examples. The Modelling and Simulation community has a variety of Standards Groups, each of which must work together to design, propose, and formally approve sets of standards. Standards Group members are drawn from organisations all over the world and must represent the interests of their members in developing the standards. Members hold regular face-to-face meetings, but use TCBWorks to plan meeting agendas, and discuss issues outside of the face-to-face meetings to reduce the need for meetings. Several U.S. Air Force bases are also using the software to support distributed teams whose members are drawn from different sections within the same base (again, an intranet). In this cases, members also hold regular face-to-face meetings, but use the software to extend the meetings. The key contributions for these distributed project teams are the ability to extend focused discussions beyond the face-to-face meetings. Face-to-face meetings (and telephone calls) are used to co-ordinate work and clarify issues requiring "richer" discussions than is possible using the electronic media.

About a quarter of TCBWorks installations have been in special purpose decision rooms, either instead of or in addition to more powerful groupware systems designed specifically to support face-to-face meetings (e.g., GroupSystems, VisionQuest). The rationale is that TCBWorks provides a simpler user interface, enables a common tool to be used in the meeting room and on the desktop, more easily enables remote group members to participate, and costs less. Creative Loafing, an Atlanta-based "alternative" newspaper with circulation of 180,000 uses TCBWorks in their decision room for strategic and operational planning, and employee 
feedback. In the next few months, the system will be opened to enable access from their four satellite offices outside of Atlanta, so that managers at these locations can participate in planning meetings over the Web combined with simultaneous conference telephone calls. Another application area that is somewhat surprising to us is the use of the software in education. About a quarter of the users are using the software to support education, either distance education across the Internet or to support class discussions in decision rooms or outside of regular class time.

\subsection{ISWorld Net Example}

The ISWorld Net example which is introduced here, is an interesting look at how TCBWorks was used by its Executive Operative Committee as a mechanism for distributed decision making. ISWorld Net (http://www.isworld.org/isworld.html) is a worldwide virtual organization and its mission is to "provide information management scholars with a single entry point to resources related to information systems technology and to promote the development of an international information infrastructure that will dramatically improve the world's ability to use information systems for creating, disseminating, and applying knowledge". Some of its founders include the Academy of Managements' Organization Communications and Information Systems Division, the Association for Information Systems, the International Conference on Information Systems and the International Federation for Information Processing: Technical Committee 8.

The members of this committee are individuals who are actively involved in helping to build and/or promote ISWorld Net. Each member is involved in developing a set of web pages to support the world-wide community of information systems faculty, with each member typically managing and developing one sub-area (e.g., DSS, groupware etc.). The committee as a whole develops strategic objectives, and operating standards, and also advise on a day-today basis the chairperson and, through him or her, the officers of the ISWorld Net Governance Committee. Because members of this committee are individuals who represent their own institutions all over the world, and their contributions are entirely voluntary, the committee requires a communication medium which is accessible to all its members to communicate and coordinate their activities. Using e-mail via setting up discussion lists was the best choice under such circumstances since most universities would have e-mail facilities.

However, when it comes to ocassions for decision making, the committee has been faced with a lack of a proper mechanism to facilitate this type of distributed virtual organizational decision making. Also, the bigger problem is the actual lack of a formal decision making process within this committee. Revisiting Habermas' (1990) theory of Communicative Action, due to the distributed nature of the committee's members, the "turn taking" (Bannon, 1994) more often is mistimed, the discussion may not be in the right sequence, and not everyone who wants to contribute can have equal time and opportunity to participate in this process. Often, in order to push through certain decisions, the chairperson will adopt the policy that no response means no objection, and will be accepted after a predetermined time has elapsed. Clearly, there is a need to address the members' and the committee's communicative competence in this respect.

Decisions to be made range from strategic decisions such as the choice of marketing strategy to promote ISWorld Net to the more mundane and time consuming discussion about 
design guidelines for page formatting. And using the email is deficient in supporting such decision making activities. The availability and accessibility of TCBWorks to this committee was welcomed and on the outset, looked very promising to suit its needs. By far, the biggest advantage is that there is little set-up time, little maintenance and all its members who are already active on the Web can use their web browsers to use this tool. A few years earlier, this scenario would not have been possible as proprietary groupware software would have been cost prohibitive and to get the client software to the distributed members (each with their different platform and operating system) would almost be an unsurmountable challenge.

Over a two-week period, the committee actively experimented with this tool. As the material is to be organized by projects, the chairperson set up a number of projects, and the members contributed the topics of interest in the various projects. Using TCBWorks, comments on each topics are archived in the sequence that they are posted and can be viewed by everyone. This is a particularly useful feature as it created the shared "collective memory" of the committee. Most members who used it genuinely felt that it has a lot of potential and promise. Also, this feature can add value by letting others see the issues that are debated (if necessary).

However, since this initial two-week period, the committee had not used TCBWorks. One of the authors of this paper sent an email to this committee to solicit for feedback about its use, with which the following replies were received:

- the committee as a whole did not really give it much of a test, maybe we should try again

- the tool did not fit the way the committee operates

- the committee does not have that many issues and when they do, email will suffice

- getting into it requires about 5 steps (what page is it on, what is the password, what is the account name, how does this work?)

- not everyone participated

The responses revealed a certain degree of expectation of TCBWorks (in comparison with other groupware products) in terms of its deficiency in interface design, interaction and ease of use. It did not however, reveal the greater problem of a lack of a decision making process. TCBWorks neccessitates that the "discussion agendas are clearly defined by the project leaders who shape and guide the discussions to reach the project objectives. Objectives and timetables are clearly defined and work assigned to various members". Though TCBWorks requires no facilitators, facilitation is still neccessary via the project leaders to make sure that participants understand and observe the requirements and constraints. In the case of the ISWorld Net committee, it was a "free for all" situation. Of the several projects that were set up, each project never achieved the critical mass of getting all the members to be involved, or the topics to be debated and decided upon.

There was a successful outcome though, on the project that was set up to test the tool. The chairperson had suggested that everyone should at least post a comment under this project, and as a result, generated the most comments. One of the discussion topic was on the choice of day to meet at the ICIS conference in Amsterdam in 1995. Another topic was to vote on this. Everyone did meet up with each other at the conference on the right day. Ironically, this date also coincided with the last use of this tool. There was no momentum to use TCBWorks again. 
This brief example of the use of TCBWorks by the ISWorld Net committee highlights both the failure and the potential of its use. The failure can be seen in terms of being an example of a good technological support for a non-relevant task at hand. The committee lacks a decision making process in the first place, and therefore, to introduce a decision making mechanism like TCBWorks will not help the situation. The potential can be seen in terms of helping to improve the communicative competence of distributed groups or communities. If there is a process, if everyone participates, if everyone who want to participate can participate, then TCBWorks stands as a good prospect of achieving just that. Already the ISWorld Net committee is considering having another go, this time with a decision making process in place.

\section{LESSONS LEARNT}

We learned a host of lessons from developing and using the system, both technical and userbased. The first major technical lesson we learned was that it is possible to develop a major application system using the Web. The use of a Web browser such as Netscape as the client environment offers some distinct advantages and disadvantages over traditional system development.

First, because the Web browser provides many built-in functions, development proceeds much faster than it would in a traditional development environment. Traditionally, $80 \%$ of any DSS is the user interface. With Web-based development, much of the interface is automatically provided by the browser; you only need to specify what functions to use. It is very much like building with children's blocks -- or objects. Interface coding is reduced to about $40 \%$ of the application, saving a significant amount of programming time. The disadvantage is that you are limited to the capabilities of the browser. For example, traditional interface concepts such as drag-and-drop are not (yet) available, so you cannot use them.

A second technical lesson was security; security concerns were expressed by a significant percentage of our users. One level of security is in the web server itself. Most servers can restrict access to only certain Internet addresses, (e.g., within one company or set of companies). The problem occurs if users regularly use CompuServe or other commercial access provider, because one must grant access to all users of those services -- not a very satisfactory solution. Using the security built into the web server was not as good an option as we initially believed. To make the software more secure, every time a user successfully logs in with a correct userid and password, the user is assigned a randomly generated authentication code that is valid for at most 12 hours, without it no access is granted. This prevents the editing scenario described above. It also prevents someone from placing a bookmark in the middle of the system to bypass the login process. You can still bookmark a screen, but the authentication code granting access will be useless after 12 hours, and the system will require you to login. The system also has a logout option, which removes the authentication code from the system and prevents anyone walking by your computer from using the Go Back option to gain unauthorised access to the system. However, logging out is not normally expected of Web-based systems, so this may not be as secure as we would like.

Learning about the software, and making an informed decision about its fit with a specific organization's needs is simplified by using the Web. Since it is Web-based, anyone with a Web 
browser can experiment with it. About 20 organizations have established databases on our server at Georgia as a trial period before deciding whether to install it on their server.

Distribution and installation on a remote server is relatively straightforward. TCBWorks and the web server and database server can be downloaded from the Web via FTP or a Web browser. The rollout of the second version of the software was rather simple from a technical perspective, illustrating one of the benefits of the Web. To upgrade the approximately 20 organizations using databases on our server, we simply added a link from the home page of version 1.0 to the home page of version 1.1. No changes were required to any client computers. Users simply added a bookmark to the new version to their browser. Likewise, the organizations using our software on their servers simply downloaded the software to a new directory and added a link from their home page.

We also learned several important lessons about user expectations. Most users have been quite enthusiastic about the software. It is a novel Web application and also provides clear value to their work groups and project teams. However, some users have been less than enthusiastic. Rather than viewing the system as a Web application, they see it as a regular desktop application and hold it to the same standards. They expect to use all the standard interface concepts such as double-clicking to open, dragging-and-dropping to move and using pull-down menus with multiple windows. These simple operations are beyond the capabilities of DSS built with today's Web browsers. Until the next generation of browsers become more common, we will not be able to satisfy users with these needs.

A second issue deals with response time. In general, every request issued by the user results in a request sent to the Web server. Response times vary depending upon network traffic. Though most intranet users have reported response times of 1-2 seconds, or less, it varies tremendously across the Internet. North American and Asian users have reported response times of 1-2 seconds, but most response times are considerably longer. Even moderately longer response times (3-4 seconds) can prove extremely frustrating, because they occur after every command. Several potential users have discontinued use of the software because the response times proved too frustrating.

Depending upon your viewpoint, the limitations of bandwidth and response times will either be a fundamental issue, or a minor one. Bandwidth will grow rapidly as new technologies are deployed (e.g., wavelength division multiplexing across fibre optic cable). However, demand will also grow as more users join the Internet and as existing users place more demand from new applications. Predicting effects becomes difficult, although we anticipate that supply will rise to just keep pace with demand, making bandwidth a continuing issue.

Finally, the experiences gained from understanding the use of TCBWorks, particularly examples like the ISWorld Net committee's use, can provide invaluable insight into justifying an orientation towards research on this new environment of distributed work and distributed collaborators, on understanding the task to be performed and less on "homogeneous groups" type research. 


\section{CONCLUSIONS}

Much of our present groupware research has focused on the one-time use of groupware for special organisational decision-making or in laboratory settings with student groups. We have far less field-based research with on-going project teams and task forces, and far less with distributed teams and task forces. Understanding the long-term effects of the use of webbased groupware becomes critical.

One key issue may centre on the role of a facilitator, a specially trained individual who assists the group in using the technology to make decisions. Current LAN-based groupware designed to support decision making in decision rooms tend to require a facilitator (e.g., GroupSystems). These system are typically complex and are designed around the presumption of the facilitator to minimise the amount of effort that regular participants must take to learn the software (Nunamaker, et al. 1991).

TCBWorks takes a different approach. It is designed to provide an extremely simple user interface that requires no facilitator. The trade-off, of course, is that it lacks some of the functionality of the complex systems. Several of the organisations now using TCBWorks in decision rooms no longer use facilitators. The facilitator was important in the initial few times that TCBWorks was used, to help in training the users on both the software and good groupware-supported decision process, but now that users have some experience, they no longer want the facilitator. The question is whether we will see this trend continue. With hindsight, this may an obvious outcome. Single-user DSS does not require facilitators, so why should group DSS? And if single-user DSS had required the use of the facilitator, would their use be cost effective, and would they have enjoyed the rather widespread use they receive today?

A limiting factor in the use of web-based groupware is the user interface. The interface is limited by the use of web-browsers, which place huge constraints on what is and is not possible. One potential solution to some of the user interface issues may be Java, although its ultimate value is unclear at this point. With Java, one can write mini-programs that could provide many of the interface characteristics desired by users, and also enable the user to work isolated from the server and only connect periodically (e.g., every few minutes) to issue updates to and receive updates from the server. Java may also permit background work, so that updates can be sent immediately, but the browser does not pause and lock the user from continuing to work. Also, we could explore web initiatives such as ISWorld Net which can provide a platform for experimentation and research. We could see how TCBWorks works for the committee the next time round after learning from their earlier experience which was not a particularly good test of it due to a lack of intellectual involvement.

We believe that we are the edge of a revolution in DSS development. We expect that within two years, most companies will realise that the Web is not only a means of electronic publishing, but can be a full-scale system development environment. Rather than developing systems for Windows, UNIX, or Apple, companies will begin developing for the Web. Even systems that are designed solely for internal use (and do not access the Internet) will use Web browsers as the client. Of course, the Web will need to mature, and there will still be the need for traditional DSS applications, but more and more applications that require networking will use the Web, rather than proprietary operating systems and networks. Those who get to the Web first, learn its intricacies, and push its limits will have a distinct advantage. But we must 
remember the context of the application as shown in the ISWorld Net example. We do not want to end up with having sophisticated systems and tools looking for decisions to make and problems to solve.

\section{REFERENCES}

Alter, S (1979) Decision Support Systems; Current Practice and Continuing Challenges. Addision-Wesley, Reading, Massachusetts.

Bannon, L.J (1994) CSCW - Challenging (G)DSS Perspectives on the role of Decisions, Information, and Technology in Organizations in B.Mayon-White, S.Ayestaran and P.Humphreys (Eds), Decision Support in Organizational Transformation. San Sebastian. Universidad del Pais Vasco Press.

Benamati, J., Lederer, A.L. and Singh M. (1995) The Impact of Rapid Change in Technology on the Information Systems Organization, in Proceedings of the First Americas Conference on Information Systems, August 25-27, Pittsburgh.

Bhargava, H.K., Krishnan, R. and Muller, R. (1995) On Sharing Decision Technologies over a Global Network, in Proceedings of the First Americas Conference on Information Systems, August 25-27, Pittsburgh.

Crawford, A. (1982) Corporate Electronic Mail - A Communication-Intensive Application of Information Technology. MIS Quarterly, 6, 1-13.

Daellenbach, H.G. (1994) Systems and Decision Making: A Management Science Approach. John Wiley and Sons, Chichester, 189.

Danyi, P. (1994) Groupware '94 Conference and Exhibition.

Dase, M.A., Tung, L. and Turban, E. (1995) A Proposed Research Framework for Distributed Group Support Systems, in Proceedings of the 28th Annual Hawaii International Conference on System Sciences, IEEE Computer Society Press, Los Alamitos, California.

Dennis,A.R. (1995) Groupware Central, http://www.cba.uga.edu/groupware/groupware.html

Dennis, A.R. and Nunamaker, J..F., and Vogel, D.R. (1990/91) A Comparison of laboratory and field research in the study of electronic meeting systems. Journal of Management Information Systems, 7 (3), Winter, 107-135.

Dennis, A.R, and Nunamaker et al (1988) Information Technology to support electronic meetings. MIS Quarterly, December, 591-624.

DeSanctis, G. and Dickson, G.W. (1987) GDSS software as "shell" system in support of a programme of research. In Proc. of 20th annual Hawaii conference on systems sciences.

Fellers, J.W., Clifton, A. and Handley, H. (1995) Using the Internet to Provide Support for Distributed Interactions, in Proceedings of the 28th Annual Hawaii International Conference on System Sciences, IEEE Computer Society Press, Los Alamitos, CA.

Fenton, N. and Hill, G. (1993) Systems Construction and Analysis: A Mathematical and Logical Framework. McGraw-Hill, Maidenhead, 18.

Gallupe, R.B. and DeSanctis, G. (1988) Computer-Based Support for Group ProblemFinding: An Experimental Investigation. MIS Quarterly 12 (2).

Ginzberg, M.J. (1975) A Process Approach to Management Science Implementation. PhD dissertation, Massachusetts Institute of Technology. 
Habermas, J. (1990) The theory of communicative action. Volume 1: Reason and the rationalisation of society. Polity Press, London.

Hackman, J.R and Kaplan, R.E. (1974) Interventions into Group Process: An Approach to Improving the Effectiveness of Groups. Decision Sciences, 5, 459-480.

Humphreys, P.C. (1984) Levels of representation in structuring decision problems. Journal of Applied Systems Analysis, 11, 3-22.

Humphreys, P.C. and Nappelbaum, E.L. (1994) Structure and communications in the process of organizational change: Eastern European experience and its general relevance. B.Mayon-White, S.Ayestaran and P.Humphreys (Eds), Decision Support in Organizational Transformation. San Sebastian. Universidad del Pais Vasco Press.

Humphreys, P.C., Berkeley, D. and Jovchelovitch, S. (1995) Organizational Psychology and Psychologists in Organizations: Focus on organizational transformation. Forthcoming in the Interamerican Journal of Psychology.

Hiltz, S.R. and Turoff, M. (1992) Virtual Meetings: Computer Conferencing and Distributed Group Support, in Computer-Augmented Teamwork: A Guided Tour (ed. R.P. Bostrom, R.T. Watson and S. Kinney), Van Nostrand, Reinhold, New York.

Huber, G.P. (1984) Issues in the Design of Group Decision Support Systems. MIS Quarterly 8 ISWorld Net (1994), http://www.isworld.org/isworld.html, November 28.

Jackson, M.C. (1991) Systems methodology for the Management Sciences, Plenum, N.Y.

Jelassi, T., Klein, M.R. and Mayon-White, W.M. (1992) Decision Support Systems: Experiences and Expectations, IFIP Working Group 8.3 Conference Proceedings, NorthHolland, Amsterdam.

Johnson-Lentz, P. and Johnson-Lentz, T. (1992) The process and impacts of design choices, in Computer-Mediated Communication Systems: Status and Evaluation (ed. Kerr, E.B. and Hiltz, S.R.), Academic Press, New York.

Kelly, M.M. and Gordon,G.E. (1986) Telecommuting: How to make it work for you and your Company. Prentice-Hall, New Jersey.

Kivijarvi, H. and Zmud, R.W. (1993) DSS implementation activities, problem domain characteristics and DSS Success. European Journal of Information Systems, 2 (5).

Krishnan,R. et al (1995), Method Servers.

Krol, E. (1992) The Whole Internet User's Guide and Catalog. O'Reilly and Associates, CA.

Lederer, A.L. and Sethi, V. (1988) The implementation of strategic information system planning methodologies. MIS Quarterly, 12, 445-461.

Lee, A. (1994) Electronic Mail as a Medium for Rich Communication: An Empirical Investigation Using Hermeneutic Interpretation. MIS Quarterly, 18 (2), 143-157.

Lucas, H.C. (1976) The Implementation of Computer-Based Models. National Association of Accountants, New York.

Lyytinen, K., Maaranen, P. and Knuuttila, J. (1994) Groups are not always the same: An analysis of group behaviours in electronic meeting systems. CSCW: An International Journal, 2 (4), 263-286.

Manley, J.H. (1975) Implementation attitudes: a model and a measurement methodology. In Implementing Operations Research/Management Science (Schultz, R.L and Slevin, D.P, eds), American Elsevier, New York, 183-202.

Markus, M.L. (1994) Electronic mail as the medium of managerial choice. Organization Science, 5 (4), 502-527. 
McCaskey, M.B. (1988) The challenge of managing ambiguity and change. In L.R. Pondy, R.L. Boland, Jr and H. Thomas (Eds), Managing ambiguity and change. Wiley \& Sons, Chichester, 1-15.

McGrath, J.E. and Hollingshead, A.B. (1994) Groups Interacting with Technology: Ideas, Evidence, Issues and an Agenda. Sage Publications.

Noam, E. (1995) What then is the role of the university?. Science 13, October, 247.

Nunamaker, J.F., Dennis, A.R., Valacich, J.S., Vogel, D.R., and George, J.F. (1993) Group Support Systems Research: Experience from lab and field, in Group Support Systems: New Perspectives (ed. Jessup, L.M. and Valacich, J.S.), Macmillan Publishing Company.

Nunamaker, Jr., J.F., Dennis, A.R., Valacich, J.S., Vogel, D.R., and George, J.F. (1991) Electronic Meeting Systems to Support Group Work. Communications of the ACM, 34 .

Phillips, L.D. (1989) People-centred group decision support. In G. Doukidis, F. Land and G. Miller (eds) Knowledge based management support systems. Chichester, Wiley \& Sons.

Power, D., Bhargava, H. and Quek, F. (1994) ISWorld Net Decision Support Systems, http://power.cba.uni.edu/isworld/dss.html.

Quek, F. and Tarr, I. (1996) An Example of the Use of the WWW as a tool and environment for Research Collaboration, in IFIP Working Group 8.4 Conference Proceeding (ed. Glasson, B. et al), April, Arizona. (in press)

Rice, R. and Shook, D. (1990) Relationships of Job Categories and Organizational Levels to Use of Communication Channels, including Electronic Mail: A Meta-Analysis and Extension. Journal of Management Studies, 27, 195-229.

Schmidt, K. (1991) Riding a Tiger, or Computer Supported Cooperative Work. In L.M. Bannon and K. Schmidt (Eds) Proceedings of the 2nd European Conference on CSCW. Kluwer, Dordrecht, 1-16.

Schmidt,K. and Bannon, L. (1992) Taking CSCW Seriously: Supporting articulating work. Computer Supported Cooperative Work, 1 (1-2), 7-40.

Shonk, J.H. (1992) Team-Based Organizations. Homewood, III., Business One Irwin.

Sprague, R.H. (1980) A framework for the development of group decision support systems. MIS Quarterly 4 (4), 1-26.

Sprague, R. H and Carlson, E.D. (1982) Building Effective Decision Support Systems. Prentice-Hall, Englewood Cliffs, New Jersey.

Steeb, R. and Johnston, S.C. (1981) A computer-based interactive system for group decision making. IEEE Trans. Syst., Man, and Cybern. 11 (8), 544-552.

Stodolsky, D. (1981) Automatic mediation in group problem solving. Behav. Res. Methods Instrum. 13 (2), 235-242.

Stohr, E.A. and Konzynski, B.R. (1992) Information Systems and Decision Process, IEEE Comuter Society Press, Los Alamitos, California.

Turban, E. (1990) Decision Support and Expert Systems: Management Support Systems. Macmillan, New York.

Turoff, M. and Hiltz, S.R. (1982) Computer support systems for group versus individual decisions. IEEE Trans. Commun. 30 (1), 82-91.

Ulrich, W. (1988). Systems thinking, systems practice and practical philosophy: A programme of research. Systems Practice, 1, 137-163.

Vogel, D. and Nunamaker, J.F. (1988) Group decision support system impact: Multimethodological exploration. In Proceedings of Conference on Technology and 
Cooperative Work, J. Galegher, R. Kraut, and C. Egido, (Eds). National Science Foundation, Bell Communications, University of Arizona, Tucson.

\section{BIOGRAPHY}

Alan R. Dennis is Associate Professor of Management in the Terry College of Business at The University of Georgia. He received a Bachelor of Computer Science from Acadia University, an M.B.A. from Queen's University, and a Ph.D. in Management Information Systems from the University of Arizona. His current research interests include group brainstorming and decision making, and the design of web-based technologies to support collaborative work. His research has appeared in MIS Quarterly, Information Systems Research, Academy of Management Journal, Management Science, Communications of the ACM, Organizational Behavior and Human Decision Processes, and J. of Applied Psychology.

Freddie Quek is an Information Systems Manager at Electronic Press Ltd (UK), which is an electronic publishing arm of the Current Science Group of medical publishing company. He received a Masters of Science from the London School of Economics, and is currently pursuing his $\mathrm{Ph} . \mathrm{D}$. in Information Systems at the same institution. He is also the Professional Activities Division Editor of ISWorld Net. His current research interests include decision support systems, electronic publishing, databases and the use of web-based technologies to support collaborative work.

Sridar K. Pootheri is a Ph.D. candidate in the Dept of Mathematics at The University of Georgia. He received a Bachelor of Math from the Vivekananda College at the University of Madras, and a Masters of Math from the Ramanjuan Inst. of Advanced Study in Mathematics at the University of Madras, India. His current research interests include Graph Theory and Combinatorics and the design of web-based technologies to support collaborative work. 